\title{
Internações por estresse no Sul do Brasil
}

\author{
Hospitalizations due to stress in Southern Brazil \\ Hospitalizaciones por estrés en el Sur de Brasil
}

\author{
Ademilson José Bernardineli \\ ORCID: https://orcid.org/0000-0001-6442-1339 \\ Universidade Estadual de Maringá, Brasil \\ E-mail: adejb@hotmail.com \\ Denise Tiemi Uchida \\ ORCID: https://orcid.org/0000-0002-5681-4826 \\ Universidade Estadual de Maringá, Brasil \\ E-mail: denisetiemi13@gmail.com \\ Lincoln Luis Silva \\ ORCID: https://orcid.org/0000-0001-8445-0743 \\ Universidade Estadual de Maringá, Brasil \\ E-mail: sirlincolnls@gmail.com \\ Claudia Regina Marchiori Antunes Araújo \\ ORCID: https://orcid.org/0000-0002-7234-6604 \\ Instituto Adventista Paranaense, Brasil \\ E-mail: claudia_marchiori@hotmail.com \\ Constanza Pujals \\ ORCID: https://orcid.org/0000-0003-0057-2335 \\ Universidade Estadual de Maringá, Brasil \\ E-mail: constanza.pujals@gmail.com \\ Sandra Marisa Pelloso \\ ORCID: https://orcid.org/0000-0001-8455-6839 \\ Universidade Estadual de Maringá, Brasil \\ E-mail: smpelloso@uem.br \\ Maria Dalva Barros Carvalho \\ ORCID: https://orcid.org/0000-0002-1377-3331 \\ Universidade Estadual de Maringá, Brasil \\ E-mail: mdbcarvalho@gmail.com \\ Raíssa Bocchi Pedroso \\ ORCID: https://orcid.org/0000-0002-0076-1032 \\ Universidade Estadual de Maringá, Brasil \\ E-mail: raissap@gmail.com
}

\begin{abstract}
Resumo
Objetivo: Investigar as internações por estresse nos estados do Sul do Brasil no período de janeiro de 2009 a dezembro de 2018. Metodologia: Foram coletados registros dos arquivos do SIH/SUS (Sistema Internação Hospitalar do Sistema único de Saúde) na plataforma eletrônica do Departamento de Informática do Sistema Único de Saúde (DATASUS), referente às internações cujo diagnóstico principal contido no capítulo V do CID - 10 pertencessem à categoria F43 "Reações ao "stress" grave e transtornos de adaptação" e subcategorias. A tendência temporal foi avaliada por regressão joinpoint (versão 3.4.2.) e a análise geoespacial por meio do software Quantum Gis (QGIS), versão 2.16. Resultados: Paraná, Santa Catarina e Rio Grande do Sul apresentaram tendência decrescente com valores de Annual Percentage Change (APC) de -14.7, -6.2 e -15.8, respectivamente. O gênero feminino foi mais incidente, com exceção de 2013 e 2017. Separando as internações F43 em subcategorias, a F43.0 (Reação aguda ao "stress") foi a principal causa de internação por estresse (4.27). A análise espacial apresentou 57 municípios High-High (p<0.05). Conclusão: Os dados obtidos sugerem que com a implantação da Portaria $n^{\circ} 3.088$ de 23 de dezembro de 2011, o número de casos por internação por estresse diminuiu. $\mathrm{O}$ gênero feminino apresentou maior risco de desenvolvimento de outras doenças e de internamento decorrentes do estresse e a tendência se mostrou decrescente com maiores internações em regiões onde há o reflexo das incertezas do mercado de trabalho, bem como da precariedade das condições sociais ideais.
\end{abstract}

Palavra-chave: Saúde mental; Brasil; Estresse; Internação.

\section{Abstract}

Objective: Investigate hospitalizations due to stress in the southern states of Brazil from January 2009 to December 2018. Methodology: Records were collected from the SIH / SUS (Hospital Internment System of the Unified Health System) files on the electronic platform of the Department of Informatics of the Unified Health System (DATASUS), 
referring to hospitalizations whose main diagnosis contained in chapter V of the ICD - 10 belonged to the category F43 "Reactions to "stress " severe and adaptation disorders" and subcategories. The time trend was assessed by joinpoint regression (version 3.4.2.) And geospatial analysis using Quantum Gis software (QGIS), version 2.16. Results: Paraná, Santa Catarina and Rio Grande do Sul presented a downward trend with Annual Percentage Change (APC) values of -14.7, -6.2 and -15.8, respectively. The female gender was more incident, with the exception of 2013 and 2017. Separating F43 hospitalizations into subcategories, F43.0 (Acute reaction to "stress") was the main cause of hospitalization due to stress (4.27). The spatial analysis showed 57 High-High municipalities (p <0.05). Conclusion: The data obtained suggest that with the implementation of Ordinance No. 3,088 of December 23, 2011, the number of cases per hospitalization due to stress decreased.

Keywords: Mental health; Brazil; Stress; Hospitalization.

\section{Resumen}

Objetivo: Investigar las hospitalizaciones por estrés en los estados del sur de Brasil desde enero de 2009 a diciembre de 2018. Metodología: Se recogieran datos de registros de los archivos SIH / SUS (Sistema de Internación Hospitalaria del Sistema Único de Salud) en la plataforma electrónica del Departamento de Informática del Sistema Único de Salud (DATASUS), referentes a las hospitalizaciones cuyo diagnóstico principal contenido en el capítulo V de la CIE-10 pertenecían a la categoría F43 "Reacciones a"estrés " trastornos severos y de adaptación" y subcategorías. La tendencia temporal se evaluó mediante regresión joinpoint (versión 3.4.2.) Y el análisis geoespacial fue realizado a partir del software Quantum Gis (QGIS), versión 2.16. Resultados: Paraná, Santa Catarina y Rio Grande do Sul mostraron una tendencia a la baja con valores de Annual Percentage Change (APC) de -14,7, -6,2 y 15,8, respectivamente. El género femenino se mostró más incidente, excepto de 2013 y 2017. Separando las hospitalizaciones F43 en subcategorías, F43.0 (Reacción aguda al "estrés") fue la principal causa de hospitalización por estrés (4.27). El análisis espacial mostró 57 municipios Alto-Alto ( $\mathrm{p}<0.05$ ). Conclusión: Los datos obtenidos sugieren que con la implementación de la Ordenanza No. 3.088 del 23 de diciembre de 2011, disminuyó el número de casos por hospitalización por estrés.

Palabras clave: Salud mental; Brasil; Estrés; Hospitalización.

\section{Introdução}

De acordo com o Relatório Global emitido pela Organização Mundial da Saúde (OMS, 2017), os transtornos mentais no Brasil ocupam a quinta posição mundial e primeira posição do continente americano, e ainda segundo a Organização Pan Americana de Saúde (PAHO, 2018) distúrbios relacionados à depressão e ansiedade atinge 7,5\% e 9.3\% da população, respectivamente. Observa-se que pessoas que passaram por algum evento adverso na vida como desemprego, luto ou trauma psicológico são mais propensas a desenvolver depressão, e ela por sua vez, pode desencadear mais estresse e piorar a situação de vida da pessoa afetada e o transtorno em si (OMS, 2017).

O estresse é uma reação espontânea do organismo (não específica) frente a uma situação difícil ou excitante que exigem alguma adaptação do indivíduo, fato em que o equilíbrio do corpo é rompido para se adaptar à nova situação e se restabelecer, podendo ocorrer com qualquer pessoa, independente da raça, idade, sexo e situação socioeconômica, sendo gerado com o intuito de manter o equilíbrio fisiológico causado por um novo estímulo dado ao corpo (Freitas, Calais \& Cardoso, 2018; Lipp, 2015). Para Guimarães, et al. (2015), ansiedade e estresse passam a ser tratados como patologia quando são exagerados, descontrolados, desproporcionais em relação ao estímulo, ou até mesmo quando distorce substancialmente do estado real do indivíduo, podendo prejudicar o raciocínio e o desempenho de atividades diárias e assim, afetar a qualidade de vida.

Diante disto, autores como Stewart e Vigod (2019), destacam alguns fatores que intensificam o risco de desenvolver traumas na saúde mental, dentre eles, estão a predisposição biológica para doença mental, histórico prévio de adversidade psíquica intensidade e cronicidade do trauma e adversidade pós-trauma.

A partir desse panorama, o Ministério da Saúde (MS), por meio da Política Nacional de Saúde Mental (PNSM), propôs uma rede de serviços de saúde mental integrada e ampliada em relação à atenção às pessoas com transtornos mentais. Essa Rede de Atenção Psicossocial (RAPS), instituída pela portaria n ${ }^{\circ} 3.088$, de 23 de dezembro de 2011, possui a finalidade de criação, ampliação e articulação de pontos de atenção à saúde, organizando a rede de serviços de saúde mental, e se 
responsabilizando por acolher indivíduos com transtornos mentais, com atendimento clínico e reabilitação psicossocial (Brasil, 2016).

Portanto, ao considerar o impacto do estresse na sociedade moderna, políticas públicas referentes à saúde mental vem ganhando espaço no âmbito do Sistema único de saúde (SUS) principalmente na atenção primária, o que revela a intenção do setor público em aprimorar e ampliar as ações de atenção básica. Entretanto, são escassos os estudos que analisam as esferas físicas, mentais e sociais da população em geral, assim, compreender o grupo de risco e os fatores associados a eles são de extrema importância. Dessa forma, o objetivo deste trabalho foi investigar o perfil das internações psiquiátricas por estresse nos estados do sul do Brasil, com o intuito de verificar as variáveis sociodemográficas como, população ocupada; salário mínimo médio mensal; renda per capta menor que meio salário mínimo; índice de desenvolvimento humano (IDH) e mortalidade infantil e realizar análises de tendência e geoespacial, a fim de subsidiar políticas preventivas para a formulação de programas de rastreamento e diagnóstico precoce.

\section{Metodologia}

Trata-se de um estudo ecológico de séries temporais, utilizando-se apenas de dados secundários, referentes aos estados da região sul do Brasil, Paraná, Santa Catarina e Rio Grande do Sul.

Os dados analisados foram obtidos nos registros dos arquivos do sistema de internação hospitalar do SUS (SIH/SUS), no período de janeiro de 2009 a dezembro de 2018, pela plataforma eletrônica do Departamento de Informática do Sistema Único de Saúde (DATASUS). Foram selecionadas todas as internações cujo diagnóstico principal está contido no capítulo V da classificação internacional de doenças (CID-10) e pertencessem à categoria F43: Reações ao "stress" grave e transtornos de adaptação (F43.0 - Reação aguda ao "stress"; F43.1 - Estado de "stress" pós-traumático; F43.2 - Transtornos de adaptação; F43.8 - Outras reações ao "stress" grave; F43.9 - Reação não especificada a um "stress" grave).

Devido à multiplicidade da autorização de internação hospitalar (AIH) de uma mesma internação, foi necessário agrupar as reinternações em uma só, para isto, foram agrupadas as AIH que possuíam a mesma data de nascimento, o mesmo sexo, o mesmo município de residência e o mesmo ano de competência da AIH.

Para analisar estatisticamente os dados, foi calculado a taxa de incidência estabelecida para cada ano separadamente, mediante a divisão do número de internações por reação ao "stress" pelo número de pessoas sob risco de exposição durante o mesmo período (dados fornecidos no Instituto Brasileiro de Geografia e Estatística - IBGEa, 2018), e o valor obtido multiplicado por 100 mil habitantes.

A tendência temporal foi avaliada por regressão JOINPOINT, versão 3.4.2. Os modelos foram ajustados assumindo número diferente de JOINPOINT, de zero (tendência representada por um único segmento de reta) até três, considerando pontos de mudança na evolução temporal das taxas.

A análise espacial foi realizada utilizando a base cartográfica fornecida pelo Instituto Brasileiro de Geografia e Estatística (IBGE) (IBGEb, 2018), com interpolação e construção de mapas temáticos realizados com base no georreferenciamento dos municípios no QGIS (versão 2.16) e R studio (versão 1.1.456). A análise exploratória da região Sul do Brasil foi realizada para verificar a auto correlação espacial entre as internações de taxas de estresse por municípios. A taxa de suavização spatial empirical bayes na matriz queen wrights, foi aplicada para minimizar as variações nas taxas por cidade $\mathrm{e}$ as possíveis flutuações aleatórias derivadas das pequenas populações. Uma avaliação com a estatística Global Moran I foi aplicada para verificar a auto correlação global. Também foram realizados indicadores locais de associação espacial (LISA), para verificar a existência de aglomerados espaciais (clusters), os quais são classificados de acordo com as características da vizinhança. As correlações espaciais globais e locais foram consideradas significativas para $\mathrm{p}<0,05$. 
A realidade sociodemográfica dos municípios obtidos na análise espacial foi descrita por meio de dados socioeconômicos obtidos no site do IBGE cidades.

\section{Resultados}

Dos três estados, o Rio Grande do Sul apresentou a maior incidência (4,11/100.000 habitantes) por internações decorrentes de estresse (Figura 1) seguido do Paraná (3,62/100.000 habitantes) e Santa Catarina (2,59/100.000 habitantes). O ano de 2011 destaca-se apresentando as maiores incidências no estado do Paraná e Rio Grande do Sul (0,66/100.000 e 0,85/100.000 habitantes, respectivamente).

Figura 1. Incidência das internações decorrentes de estresse (CID 10 - F43), no três estados do Sul do Brasil, para os anos de 2009 a 2018.

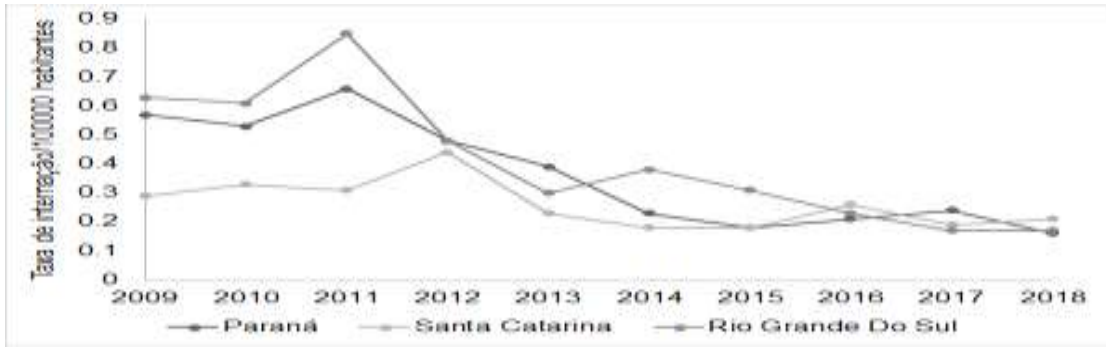

Fonte: Dados da pesquisa.

De acordo com a análise da regressão por JOINPOINT, as taxas de incidência apresentaram uma tendência significativamente decrescente para todos os estados no período estudado. O estado do Paraná apresentou um decréscimo anual (Annual Percentage Change) de 14,7\% (Figura 2A), Santa Catarina, um decréscimo de 6,2\% ao ano (Figura 2B) e Rio Grande do Sul, um decréscimo de 15,8\% ao ano (Figura 2C). 
Research, Society and Development, v. 10, n. 5, e51410515259, 2021

(CC BY 4.0) | ISSN 2525-3409 | DOI: http://dx.doi.org/10.33448/rsd-v10i5.15259

Figura 2. Tendência da incidência das internações por estresse (categoria F43: Reações ao "stress" grave e transtornos de adaptação) entre os anos de 2009 e 2018 nos três estados do Sul do Brasil

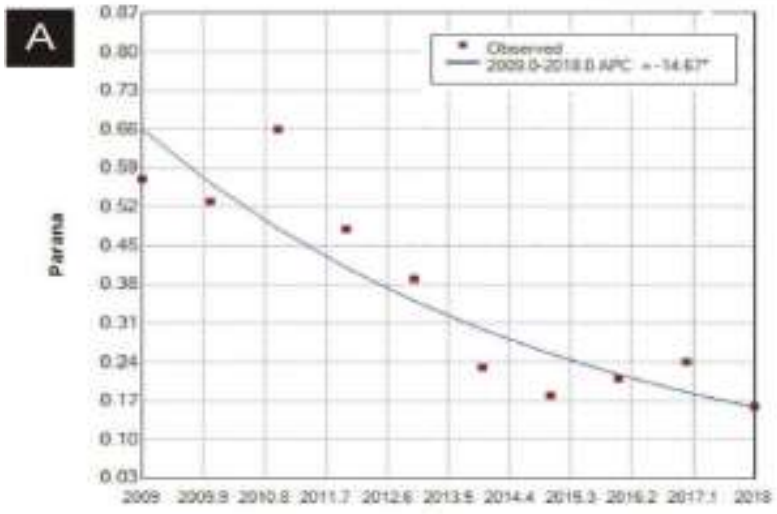

B

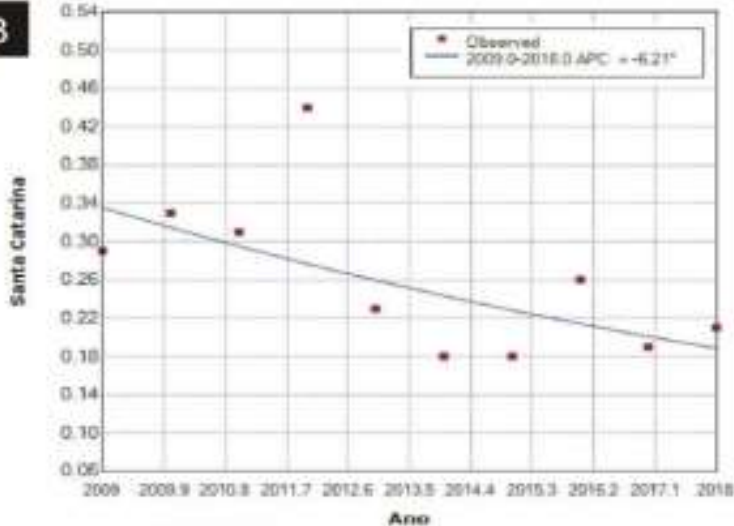

C

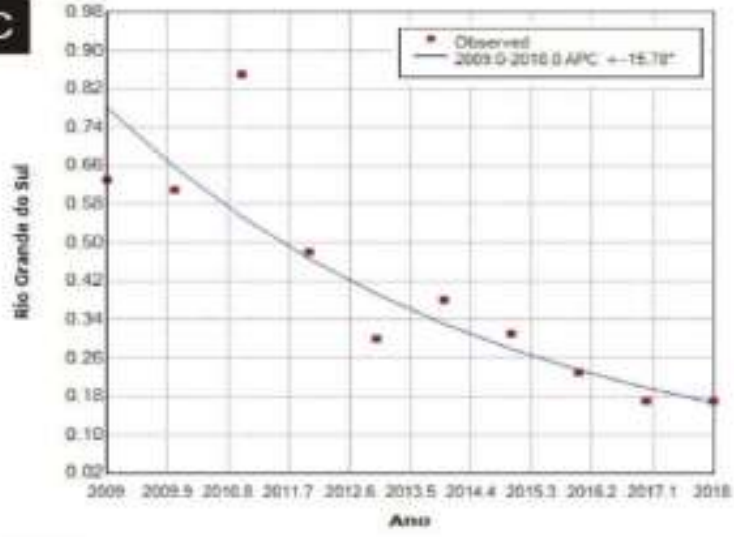

[A] Paraná, APC -14,7*; IC95\% -19,5; -9,6; [B] Santa Catarina, APC -6,21*; IC95\% -11,7; -0,3; e [C] Rio Grande do Sul APC -15,78* IC95\% -20,2; -11,1. *indica que a alteração percentual anual (APC) é significativamente diferente de zero no nível alfa $=0,05$.

Fonte: Dados da pesquisa.

Quando estratificamos as internações quanto ao sexo (Figura 3), observa-se que as mulheres tiveram maior registro de internações em todos os estados do sul do Brasil nos 10 anos avaliados (exceto para o Paraná no ano de 2017, e Santa Catarina, no ano de 2013). 
Figura 3. Taxas de internação por "stress" nos estados do sul do Brasil, segundo categoria do capítulo V do CID 10 e Taxa (por 100 mil habitantes), nos anos de 2009 - 2018.

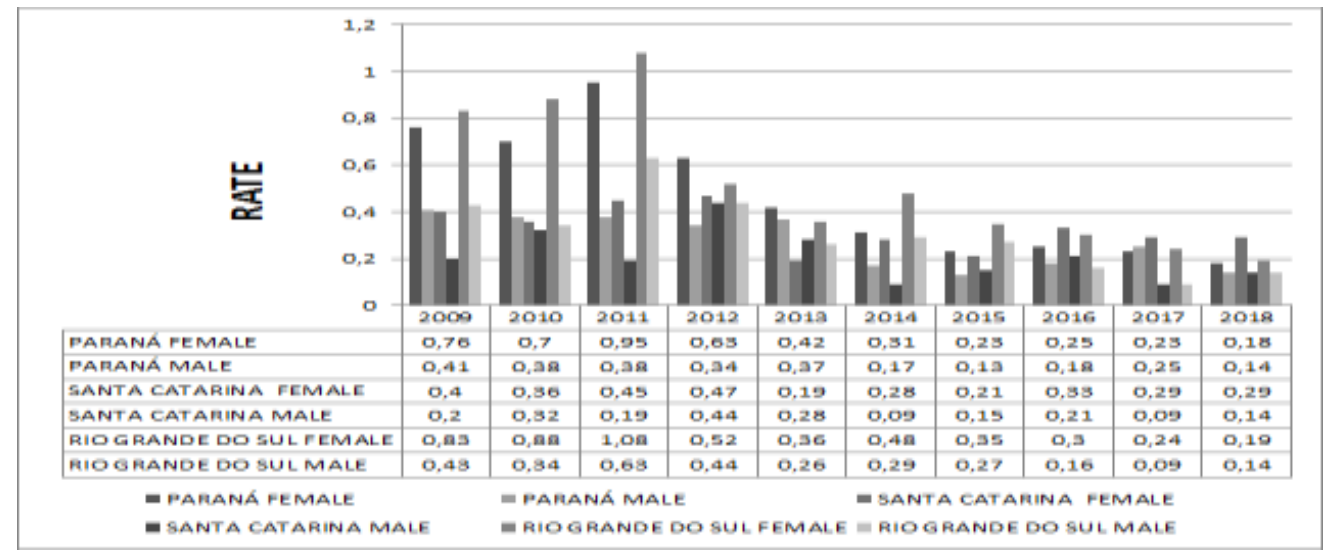

Fonte: Dados da pesquisa.

Ao avaliar as sub-categorias de F43 (F43.0 - Reação aguda ao "stress"; F43.1 - Estado de "stress" pós-traumático; F43.2 - Transtornos de adaptação; F43.8 - Outras reações ao "stress" grave; F43.9 - Reação não especificada a um "stress" grave) observou-se que F43.0 foi a principal causa de internação por estresse (4,27/100.000), seguida de F43.2 (2,54/100.000) e F43.1 (1,65/100.000). No estado do Paraná, a menor taxa de incidência se deu por F43.9 (0,20/100.000), em Santa Catarina F43.8 (0,14/100.000) e no Rio Grande do Sul F43.8 (0,16/100.000).

$\mathrm{Na}$ análise espacial, o indicador Global de Moran =0,44 (p<0,05) mostrou que a distribuição das taxas de internações por estresse no sul do Brasil apresenta formação de clusters. Para verificar a localização e a quantidade de agrupamentos formados, a análise do LISA apontou 70 municípios formaram clusters do tipo High-High (p<0,05), ou seja, municípios com altas taxas de internações por estresse também estão rodeados de municípios com alta taxa de internação (Figura 4).

Figura 4. Indicador de auto correlação espacial (LISA) das taxas de internações por estresse no sul do Brasil, 2009 - 2018.

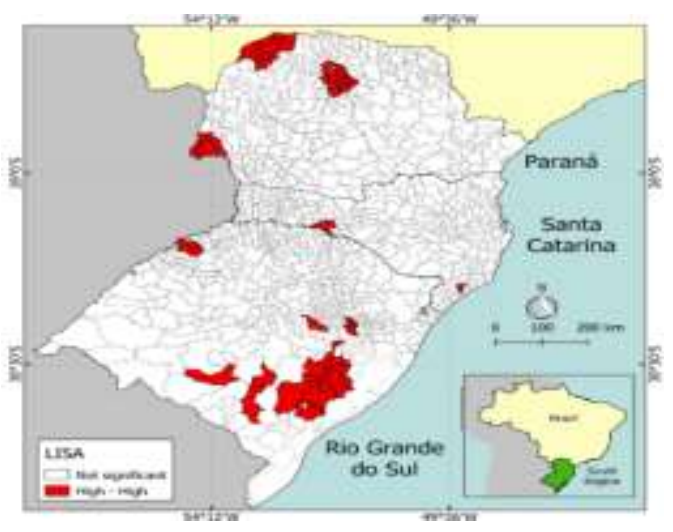

Fonte: Dados da pesquisa.

Considerando os municípios de cada região que apresentaram significância estatística na regressão geoespacial, buscou-se no site IBGE cidades, os dados socioeconômicos de cada município, e obteve-se as informações descritivas apresentadas na Tabela 1. 
Tabela 1 - descrição da média de dados socioeconômicos dos municípios que da região Sul que apresentaram significância estatística obtidos por meio de análise geoespacial comparados com a média dos mesmos dados do Brasil.

\begin{tabular}{|c|c|c|c|c|c|}
\hline & $\begin{array}{c}\text { População } \\
\text { ocupada (em } \\
\%)\end{array}$ & $\begin{array}{l}\text { Salário mínimo } \\
\text { médio mensal }\end{array}$ & $\begin{array}{c}\text { Renda per capta } \\
\text { menor que } 0,5 \\
\text { salário mínimo }(\%)\end{array}$ & IDH & $\begin{array}{c}\text { Mortalidade } \\
\text { infantil (óbitos } \\
\text { por } 1000 \text { nascidos } \\
\text { vivos) } \\
\end{array}$ \\
\hline $\begin{array}{l}\text { Municípios } \\
\text { analisados }\end{array}$ & 20,51 & 2,17 & 30,94 & 0,69 & 15,38 \\
\hline Brasil & 55,3 & 1,5 & 44,7 & 0,76 & 10,99 \\
\hline
\end{tabular}

Fonte: IBGE.

\section{Discussão}

Para Barros, Tung e Mari (2010), os serviços de emergências psiquiátricas relacionam-se com todos os serviços hospitalares e extra-hospitalares, possibilitando a organização do fluxo das internações e evitando sobrecarga da rede de saúde mental. Em nosso estudo, a análise dos dados evidenciou uma tendência decrescente nas internações por estresse no sul do Brasil, e pode ser observada a partir de 2011, refletindo o movimento de Reforma Psiquiátrica. Este período é caracterizado pela implantação de políticas públicas voltadas à saúde mental na rede de atenção primária e instituída pela portaria ${ }^{\circ} 3.088$ de 23 de dezembro de 2011 que em busca de ampliar esses serviços, o Ministério da Saúde instituiu em 2011 a Rede de Atenção Psicossocial (RAPS), estabelecendo os seguintes componentes: atenção primária, atenção psicossocial especializada, atenção de urgência e emergência, atenção residencial de caráter transitório, atenção hospitalar e estratégias de desinstitucionalização e reabilitação psicossocial (Brasil, 2011).

Esta tendência decrescente pode estar relacionada à eficácia de ações positivas em prol da saúde mental da população. Segundo Pitta (2011), o retorno positivo oriundo da implantação da portaria $n^{\circ} 3.088$, propiciou ações, auxílios e intervenções intersetoriais que deram conta de tratar dos indivíduos, de sua existência, de seus direitos e de suas condições concretas de vida. Ainda sobre esta portaria, Zanardo, Silveira, Rocha e Rocha (2017) corroboram com o autor supracitado ao afirmar que ela agrega os diferentes serviços em uma rede territorializada, incluindo desde os cuidados na atenção primária à saúde até a atenção hospitalar, reforçando sua articulação como forma de garantir a efetividade do cuidado.

Importante destacar que a referida portaria se efetiva após a mudança no modelo de assistência psicossocial que ocorreu com o marco legal da lei n.10.216/01 e prevê, dentre outros fatores, a alteração de financiamento e o progressivo fechamento dos leitos em hospitais psiquiátricos. De acordo com Pereira et al. (2014), a partir desta lei, deu-se início o movimento de retirada dos indivíduos internados por longo tempo das instituições para ocupar novamente o território da cidade. Afirmam ainda que os serviços de saúde mental em ambiente hospitalar funcionam com a lógica da desinstitucionalização, e estão dedicados aos casos mais graves. Os casos menos graves são atendidos na atenção básica ou nos serviços ambulatoriais, seguindo as mesmas diretrizes da reforma psiquiátrica. Segundo Gama (2012), a desinstitucionalização ocasionou uma diminuição rigorosa do número de leitos psiquiátricos, um maior cuidado com os direitos dos pacientes, a alteração do subsídio prioritário para os serviços abertos em substituição aos hospitais, bem como projetos de reinserção social através de geração de renda e construção cultural, visando a qualidade de vida dos pacientes com sofrimento psíquico.

Considerando a implementação da portaria $\mathrm{n}^{\mathrm{o}} 3.088$ e da lei $\mathrm{n}^{\circ} 10.216 / 2001$ e que, embora o estudo tenha mostrado tendência decrescente no número de internações por estresse, é importante estacar que devido a impactos sociais recentes que se apresentam cada vez mais estressantes e sofridos pela população, se faz emergencialmente necessário maiores investimentos na área de saúde mental, porém crises financeiras locais ou mundiais impactam substancialmente os repasses de recursos para as ações de promoção e recuperação da saúde. De acordo com a OMS (2013), as crises financeiras são um grande exemplo 
macroeconômico que leva a cortes nos financiamentos no setor da saúde, e dentre estes, estão as reduções nas políticas públicas voltadas à saúde mental, apesar da necessidade cada vez mais crescente de investimentos nessa área, bem como da implementação de serviços sociais devido ao crescimento nos índices de transtornos mentais generalizados e suicídio. Acrescenta-se ainda que, em muitas sociedades, os transtornos mental relacionados à marginalização e empobrecimento, baixo índice de desenvolvimento econômico (IDH), excesso de trabalho, estresse, violência e abuso doméstico são motivos de crescente preocupação, principalmente para a saúde da mulher (OMS, 2013).

No período analisado, o total da taxa de internação foi de 8,0/100.000 habitantes para pessoas do sexo masculino e 13,36/100.000 habitantes para pessoas do sexo feminino, o que demonstra a razão de 1,67 mulheres internadas por estresse para cada homem no mesmo período, este dado ressalta que as mulheres sofrem mais por estresse que os homens. Para Zordan, Falcke e Wagner (2014) e Figueiredo e Dinis (2018), a ideia das mulheres se dedicarem apenas aos afazeres domésticos e assumirem o papel de mães e esposas vêm sofrendo transformações nas últimas décadas. Embora as mulheres sempre tenham trabalhado, os movimentos sociais que surgiram nas décadas do século XX possibilitaram-nas de adentrar na carreira profissional. E ainda, para Costa (2018), as mulheres sentem-se muito culpadas e cansadas, uma vez que, por buscarem um espaço fora do lar e não terem com quem dividir as tarefas domésticas, cuidado dos filhos, a sobrecarga e o acúmulo das funções se torna evidente. Com intuito de obter uma vida melhor para si e sua família e as oscilações econômicas causadas por crises financeiras históricas, levam as mulheres a trabalharem, e na maioria das vezes não recebem apoio de seus cônjuges e familiares, e por vergonha de mostrar fraqueza, a falta de conhecimento ou a falta de tempo faz com que não busquem ajuda psicológica.

Em um estudo realizado na Índia, onde analisaram os fatores estressores da vida e os fatores atenuantes de mães que trabalham, Travasso, Rajaraman e Heymann (2014), constataram que as consequências do estresse relatado pelas entrevistadas incluíam incapacidade de cuidar adequadamente da família, ter que bater nas crianças, mau desempenho no trabalho ou ausência total de trabalho, brigar com cônjuge e familiares além da falta de desejo de interagir com outras pessoas, os pesquisadores ainda verificaram que nenhuma das participantes mencionaram buscar ajuda profissional psicológica.

Este estresse vivido no cotidiano da mulher pode levá-la a desenvolver doenças como depressão, ansiedade, doenças cardíacas, dentre outras. Em pesquisa da Sociedade de Cardiologia do Estado de São Paulo (SOCESP, 2019), 51,6\% das mulheres entrevistadas passaram por, pelo menos, um fator de estresse muito importante no último ano. Já os homens nessa situação foram $37,3 \%$. Os autores relatam a relação entre doenças do coração e saúde mental, esclarecendo que mundialmente, essas enfermidades são culpadas pelo aumento de duas a cinco vezes as chances de infarto e Acidente Vascular Encefálico (AVE). Já no Brasil, a presença do estresse agrava em oito vezes as chances de uma brasileira enfartar, sendo quase o dobro de países como a Argentina e o quádruplo da Colômbia.

Os municípios apontados na análise geoespacial, juntamente com seus dados socioeconômicos (tabela 1), refletem a realidade do estresse na região. Constatamos que, para a maioria das cidades apontadas, a atividade econômica prevalente é a agropecuária e serviços relacionados à atividade rural, que por condições ambientais geram incertezas quanto à produtividade, acentuando características do estresse, além disso, a baixa taxa de ocupação mostra um cenário de informalidade que reflete as incertezas do mercado de trabalho da maioria da população. Para Peres, Silva e carvalho (2003), a ausência de emprego, além de inviabilizar economicamente a sobrevivência do sujeito, causa uma sensação de falta de identidade capaz de pôr em risco seu equilíbrio psíquico.

A quantidade de pessoas que vivem com meio salário mínimo mensal ou menos, que caracteriza a linha de pobreza e as baixas médias de salário mínimo mensal, concomitantemente com os valores do IDH das cidades analisadas, corroboram para elucidar as diferenças de renda e a desigualdade social vivida nessas regiões, motivo este que elevam o índice de estresse. Rasella, et al. (2018) analisaram os impactos da crise econômica e as medidas de austeridade do Brasil desde 2014, afirmam 
que uma recessão aguda e profunda no Brasil tem se desdobrado, com contrações anual do PIB de 3,8\% e 3,6\% em 2015 e 2016, respectivamente. A crise econômica levou a um aumento do desemprego, principalmente em populações de baixa renda, e a taxa de pobreza (aqueles com uma renda de menos de US\$ 43 por mês) aumentou de 7,4\% em 2014 para 8,7\% em 2015, com a taxa de pobreza extrema (aqueles com uma renda de menos de US \$ 21 por mês) o aumento de 2,8\% para 3,4\%. Estas medidas econômicas e seus impactos sociais influenciam diretamente na vida das pessoas, contribuindo assim para elevar o nível de estresse, outro dado relevante a considerar é a mortalidade infantil, este índice mede a qualidade de vida da população de modo geral e a eficácia dos serviços de saúde. No Brasil, o IBGE aponta para um valor de 12,8 óbitos por nascidos vivos em 2017, já nas regiões estudadas, a média é 14,99, valor alto se comparado ao país. Este cenário mostra como a qualidade de vida e dos serviços públicos de saúde ainda deixam a desejar nestas localidades, contribuindo assim para o aumento dos índices de estresse.

Embora a implantação da portaria 3.088 fosse eficaz em contribuir para reduzir as internações por estresse na região Sul do Brasil, ainda há um longo caminho a percorrer, perpassando por maiores investimentos na área da saúde, principalmente no que tange à saúde mental.

\section{Conclusão}

No período de 2009 a 2018 o número de internações psiquiátricas por estresse nos estados do sul do Brasil, analisados por meio dos dados referentes à categoria F43 do CID-10, mostrou tendência decrescente. Este fato possivelmente deve-se à implantação da portaria 3.088 de 2011, que estabelece políticas voltadas para a saúde mental na rede de atenção primária. O maior número de internamentos ocorreu para pessoas do sexo feminino em todos os estados analisados, e as internações por reação aguda ao "stress" foi a causa principal de internação.

Considerando que o estresse é um fator desencadeante para outras doenças e agravos de saúde, é importante salientar que reduzir a mortalidade infantil, promover a igualdade e erradicar a pobreza extrema não podem ser alcançados sem um foco específico na saúde mental da população, principalmente das mulheres. Neste sentido, o estudo fornece informações para a busca de melhorias nas políticas públicas no que se refere à saúde mental, de modo a garantir maior eficácia e impacto positivo nesta área, porém, recomendam-se estudos que ampliam a forma como o estresse tem se estabelecido na sociedade, lançando um olhar mais específico quanto às vulnerabilidades sociais e precariedade de acesso ao sistema de saúde. Poucas publicações recentes que analisam a portaria $\mathrm{n}^{\circ} 3.088 / 11$ e a lei $\mathrm{n}^{\circ} 10.216 / 01$, e a ausência de alguns dados sociodemográficos de alguns municípios no site do IBGE foram as limitações deste estudo.

\section{Referências}

Barros, R. E. M., Tung, T. C., \& Mari, J. J. (2010). Serviços de emergência psiquiátrica e suas relações com a rede de saúde mental Brasileira. Brazilian Journal of Psychiatry, 32(2), S71-S77. https://doi.org/10.1590/S1516-44462010000600003

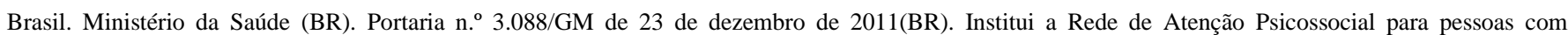
sofrimento ou transtorno mental e com necessidades decorrentes do uso de crack, álcool e outras drogas, no âmbito do Sistema Único de Saúde (SUS). Diário Oficial da União, Brasília (DF), 26 dez 2011. 19

Brasil. Ministério da Saúde (BR). Secretaria de Atenção à Saúde. Departamento de Atenção Básica. Núcleo de Apoio à Saúde da Família / Ministério da Saúde, Secretaria de Atenção à Saúde, Departamento de Atenção Básica. Brasília: Ministério da Saúde, 2014. 116 p.

Brasil. Lei $\mathrm{n}^{\circ}$ 10.216, de 6 de abril de 2001. Dispõe sobre a proteção e os direitos das pessoas portadoras de transtornos mentais e redireciona o modelo assistencial em saúde mental. Diário Oficial Eletrônico, Brasília, DF, 09 abr. 2001, p. 2.

Costa, F. A. da. (2018). Mulher, trabalho e família: os impactos do trabalho na subjetividade da mulher e em suas relações familiares. Pretextos - Revista Da Graduação Em Psicologia Da PUC Minas, 3(6), 434 -452. Recuperado de http://periodicos.pucminas.br/index.php/pretextos/article/view/15986

Figueiredo, M. G. de, \& Diniz, G. R. S. (2018). Mulheres, casamento e carreira: um olhar sob a perspectiva sistêmica feminista. Nova Perspectiva Sistêmica, 27(60), 100-119. https://doi.org/10.38034/nps.v27i60.393 
Freitas, G. R., Calais, S. L. \& Cardoso, H. F. (2018). Estresse, ansiedade e qualidade de vida em professores: efeitos do relaxamento progressivo. Psicologia Escolar e Educacional, 22(2), 319-326. https://doi.org/10.1590/2175-35392018018180

Gama, J. R. de A. (2012). A reforma psiquiátrica e seus críticos: considerações sobre a noção de doença mental e seus efeitos assistenciais. Physis: Revista de Saúde Coletiva, 22(4), 1397-1417. https://doi.org/10.1590/S0103-73312012000400008

Guimarães, A. M. V., Neto, A. C. da S., Vilar, A. T. S., Almeida, B. G. da C., Fermoseli, A. F. de O. \& Albuquerque, C. M. F. de. (2015). Transtornos De Ansiedade: Um Estudo De Prevalência Sobre As Fobias Específicas E A Importância Da Ajuda PsicológicA. Caderno De Graduação - Ciências Biológicas E Da Saúde. 3(1), 115-128. https://periodicos.set.edu.br/fitsbiosaude/article/view/2611

Instituto Brasileiro De Geografia E Estatística (IBGE cidades). <https://cidades.ibge.gov.br/>.

Instituto Brasileiro De Geografia E Estatística (IBGEa). Projeções da população, 2018. https://www.ibge.gov.br/estatisticas/sociais/populacao/9103estimativas-de-populacao.html?=\&t=o-que-e

Instituto Brasileiro De Geografia E Estatística (IBGEb). Bases cartográficas, 2018. <https://mapas.ibge.gov.br/bases-e-referenciais/basescartograficas/cartas.html>.

Lipp, M. E. N. (2015). Manual do inventário de sintomas de stress para adultos de Lipp (ISSL). (3a ed.), Casa do Psicólogo.

Organização Mundial Da Saúde (OMS). Depression and Other Common Mental Disorders. $2017 . \quad<$ https://apps.who.int/iris/bitstream/hand le/10665/254610/WHO-MSD-MER-2017.2-eng.pdf;jsessionid=848D873D4B3BE7A7 2CA6F00CCA9A0954? sequence=1 > .

Organização Mundial Da Saúde (OMS). Plan de acción sobre salud mental 2013-2020 http://www.who.int/mental_health/publications/action_plan/es/ .

Organização Pan-Americana da Saúde. The Burden of Mental Disorders in the Region of the Americas, 2018. OPAS.

Pereira, R. A. S., Silva, M. B., Souza, L. V., Araujo, A. M. de O., Costa, R. de A. da, \& Silva, F. G. d, (2014). O processo de desinstitucionalização no Brasil. cliniCAPS, 8(23). https://www.clinicaps.com.br/Pereira_R_A_S_\&_Cols.pdf

Peres, R. S., Silva, J. A. da, \& Carvalho, A. M. R. de. (2003). Um olhar psicológico acerca do desemprego e da precariedade das relações de trabalho. Psicologia: teoria $\quad$ prática, 5(1), 97-110. http://pepsic.bvsalud.org/scielo.php?script=sci_arttext\&pid=S1516$36872003000100008 \& \operatorname{lng}=$ pt\&tlng=pt.

Pitta, A. M. F. (2011). Um balanço da reforma psiquiátrica brasileira: instituições, atores e políticas. Ciência \& Saúde Coletiva, 16(12), 45794589. https://doi.org/10.1590/S1413-81232011001300002

Rasella, D., Basu, S., Hone, T., Souza, R. P., Reis, C. O. O., \& Millett, C. (2018). Child morbidity and mortality associated with alternative policy responses to the economic crisis in Brazil: A nationwide microsimulation study. PLoS Med 15 (5): e1002570.

Sociedade De Cardiologia Do Estado De São Paulo (SOCESP). Saúde cardiovascular da mulher brasileira. São Paulo, Brasil, 2019. <http://mulherespelocoracao.com.br/wp-content/uploads/2019/09/Saude-Cardiovascular-das-Mulheres-SOCESP-Final-version-24-09-19-SOCESP_HF.pdf>.

Stewart, D. E., \& Vigod, S. N. (2019). Update on Mental Health Aspects of Intimate Partner Violence. The Medical clinics of North America, 103(4), 735749. https://doi.org/10.1016/j.mcna.2019.02.010

Travasso, S. M., Rajaraman, D., \& Heymann, S. J. (2014). A qualitative study of factors affecting mental health amongst low-income working mothers in Bangalore, India. BMC women's health, 14, 22. https://doi.org/10.1186/1472-6874-14-22

Zanardo, G. L. de P., Silveira, L. H. de C., Rocha, C. M. F., \& Rocha, K. B. (2017). Internações e reinternações psiquiátricas em um hospital geral de Porto Alegre: características sociodemográficas, clínicas e do uso da Rede de Atenção Psicossocial. Revista Brasileira de Epidemiologia,20(3), 460474. https://doi.org/10.1590/1980-5497201700030009

Zordan, E. P., Falcke, D., \& Wagner, A. (2014). Copiar ou (re)criar? Perspectivas histórico-contextuais do casamento. In A. Wagner (Org.), Como se perpetua a família? A transmissão dos modelos familiares. EdiPUCRS, 47-65. 